\title{
LEARNING CURVE FOR ULTRASOUND GUIDED REGIONAL ANESTHESIA
}

Mazen Al-Sohaibani MD, Lawrence Lou MD, Ban CH Tsui MSc MD FRCPC

Department of Anesthesiology and Pain Medicine, University of Alberta, 8-120 Clinical

Sciences Building, Edmonton, Alberta, T6G 2G3

\section{INTRODUCTION}

The use of ultrasound (U/S) for regional anesthesia is an emerging and important field in anesthesiology. Substantial time and effort must be devoted in order to learn how to use $\mathrm{U} / \mathrm{S}$ to obtain a suitable anatomic image efficiently. We hypothesize that some blocks have a prolonged learning curve than others and may not be suitable for inexperienced clinicians. This study examined residents' ability to learn and use U/S to identify the brachial plexus from an interscalene and supraclavicular approach.

\section{METHODS}

Following IRB approval, 9 anesthesiology residents (PGY1-4) were given a twentyminute presentation on the use of U/S for regional anesthesia and practiced finding the brachial plexus using an interscalene and a supraclavicular approach. Up to 5 minutes were allowed to find the brachial plexus image using each approach. Eight weeks later the residents were randomly assigned sealed envelopes that contained instructions to either find the brachial plexus with these same two approaches. Each resident was allowed up to 5 minutes to read the instructions and a maximum of 5 minutes to obtain the clearest U/S picture of the brachial plexus on a volunteer. Time to find the correct image and the number of attempts were recorded. Each resident was then given instructions for the alternate approach. The procedure was then repeated using the alternate technique. The same volunteer was used for all 9 residents. The same qualified anesthesiologist judged U/S image accuracy.

\section{RESULTS}

\begin{tabular}{|c|c|c|c|c|c|}
\hline \multicolumn{3}{|c|}{ Interscalene Approach } & \multicolumn{3}{c|}{ Supraclavicular Approach } \\
\hline Time (SD) & Attempts (SD) & $\%$ Success & Time (SD) & Attempts (SD) & $\%$ Success \\
\hline $270.2(83.9)$ & $1.9(1.1)$ & 22 & $44.7(25.8)^{*}$ & $1.0(0.0)$ & $100^{*}$ \\
\hline
\end{tabular}

\section{DISCUSSION}

The time it took the residents' to identify the brachial plexus with U/S was significantly shorter using a supraclavicular approach. In addition, using a supraclavicular approach, the brachial plexus was identified $100 \%$ of the time compared to only $22 \%$ with the interscalene approach. This may be because using the supraclavicular approach the brachial plexus is viewed in close proximity to a readily identifiable vascular structure (i.e. the subclavian artery). A supraclavicular approach with U/S may be more suitable for inexperienced clinicians when identifying the brachial plexus image as it appears to be more reliable. 